\title{
The RAP Index during Intracranial Pressure Monitoring as a Clinical Guiding for Surgically Treated Aneurysmal Subarachnoid Hemorrhage: Consecutive Series of Single Surgeon
}

\author{
Sung-Chul Jin, Byung Sam Choi, Jung-Soo Kim \\ Department of Neurosurgery, Inje University Haeundae Paik Hospital, Busan, Korea
}

Background: It is well known that assessing the RAP index along with intracranial pressure (ICP) monitoring in traumatic brain injury patients is helpful. We will discuss the usefulness of this assessment tool as a clinical guide for surgically treated poor grade aneurysmal subarachnoid hemorrhage (SAH).

Methods: This retrospective study included 35 patients with aneurysmal SAH who presented with World Federation of Neurosurgical Societies (WFNS) grade V SAH and received surgical treatment from January 2013 to December 2018. Emergency surgical clipping, hematoma removal, extraventricular drainage, and if needed, wide decompressive craniectomy were combined as the proper surgical treatments. Outcomes were assessed based on in-hospital survival and the Glasgow outcome scale score at 14-day follow-up. We compared the mortality rate of two groups of seven patients: ICP monitoring only $(n=5)$ and ICP monitoring combined with the RAP index $(n=2)$.

Results: The in-hospital 14-day mortality rate by brain lesion was $48.5 \%(n=17)$. Seven patients had real-time ICP monitoring. Before 2018, three of five patients with poor WFNS grade who received real-time ICP monitoring only died. There were no deaths in the group of two patients receiving real-time ICP monitoring and the RAP index.

Conclusions: Our data indicate that combining the RAP index and ICP monitoring can be used as markers for critical intracranial physiological parameters in poor grade WFNS patients.

Key Words: cerebrospinal fluid; intracranial aneurysm; intracranial pressure; subarachnoid hemorrhage

\section{INTRODUCTION}

Patients with poor World Federation of Neurosurgical Societies (WFNS) graded aneurysmal subarachnoid hemorrhage (SAH) have high mortality rates and show poor neurological outcomes [1,2]. Early aggressive surgical clipping improves the prognoses in poor WFNS graded SAH patients. After surgical treatment, neurological examinations for these patients should be performed in a timely manner to determine if they need additional procedures. Neurological deterioration is a result of worsening intracranial conditions. If neurological deterioration occurs, sometimes the best time for appropriate treatment may already have passed. Intra-

\section{Original Article}

Received: January 21, 2019

Revised: February 15, 2019

Accepted: February 20, 2019

Corresponding author Jung-Soo Kim

Department of Neurosurgery, Inje University Haeundae Paik Hospital, 875 Haeun-daero, Haeundae-gu, Busan 48108, Korea

Tel: +82-51-797-0840

Fax: +82-51-797-0841

E-mail:jheaj@hanmail.net

Copyright () 2019 The Korean Society of Critical Care Medicine

This is an Open Access article distributed under the terms of Creative Attributions Non-Commercial License (http:// creativecommons.org/li-censes/by-nc/4.0/) which permits unrestricted noncommercial use, distribution, and reproduction in any medium, provided the original work is properly cited. 
cranial pressure (ICP) monitoring is helpful for patients who have neurologic deteriorations that are not easily detected. The ICP monitoring methods include insertion of an extraventricular drainage (EVD) catheter, a hematoma catheter [3], and diverse parenchymal and subdural ICP probes that can accommodate ICP monitoring so clinicians can take immediate action if the patient's medical condition gets worse. It is known, however, that precise monitoring of critical ICP is a challenge due to brain autoregulation that continually adjusts cerebrospinal fluid (CSF) and blood flow to maintain homeostatic conditions. Other patient comorbidities also contribute to making timely, appropriate treatments for SAH difficult. For traumatic brain injury patient management, use of the RAP index and ICP mean pulse amplitude (AMP), derived from ICP wave analysis, are quite useful in the clinical setting. They allow physicians to proactively respond to changing patient conditions before they reach critical ICP, hence mitigating irreversible damage to brain tissue and improving patient outcomes. To confirm these benefits, we studied the use of these markers for postoperative management of WFNS graded poor aneurysmal SAH patients presenting with increased perioperative ICP. Thus, this study compared two groups: real time ICP monitoring only and combined RAP index and ICP monitoring.
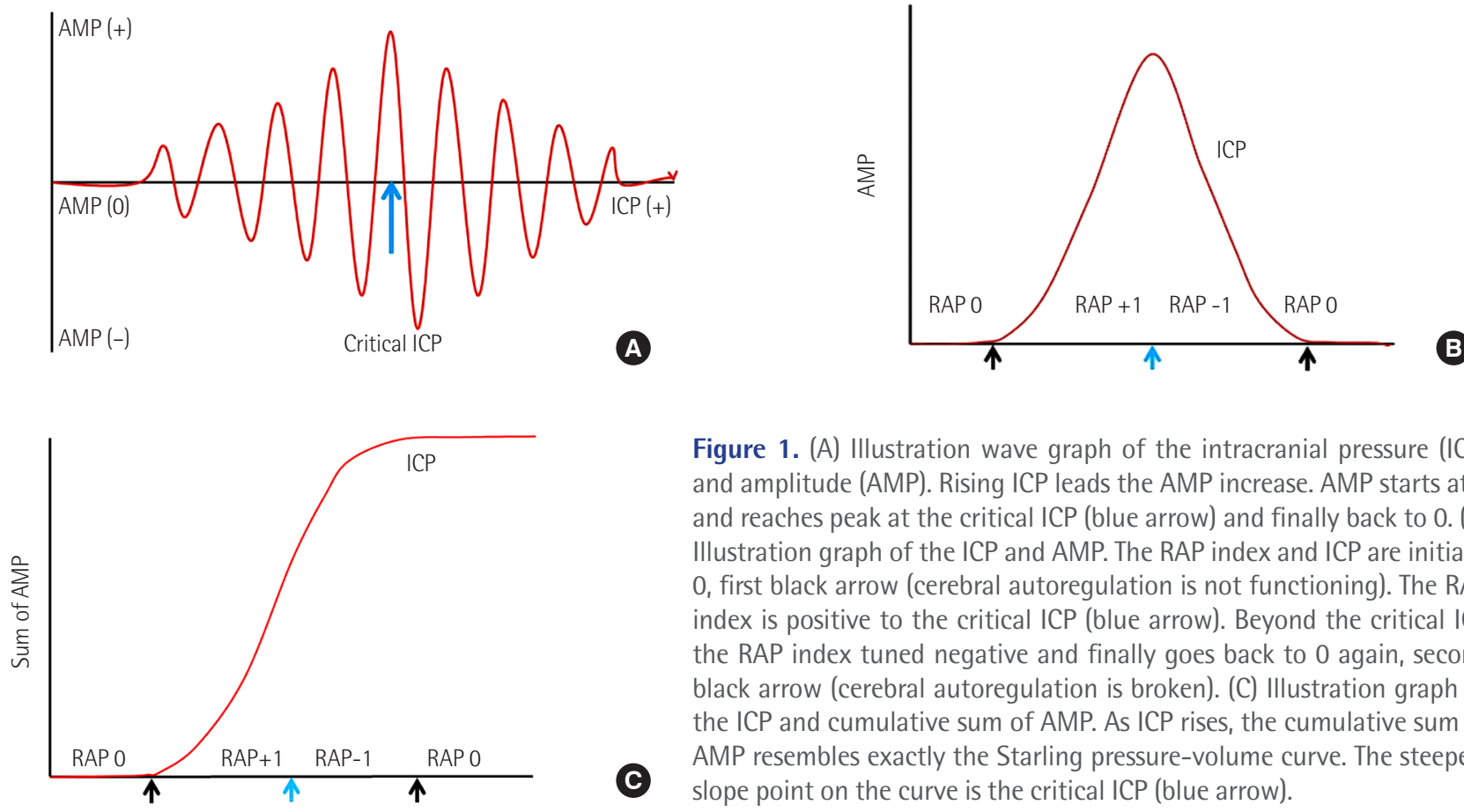

Figure 1. (A) Illustration wave graph of the intracranial pressure (ICP) and amplitude (AMP). Rising ICP leads the AMP increase. AMP starts at 0 and reaches peak at the critical ICP (blue arrow) and finally back to 0 . (B) Illustration graph of the ICP and AMP. The RAP index and ICP are initially 0 , first black arrow (cerebral autoregulation is not functioning). The RAP index is positive to the critical ICP (blue arrow). Beyond the critical ICP the RAP index tuned negative and finally goes back to 0 again, second black arrow (cerebral autoregulation is broken). (C) Illustration graph of the ICP and cumulative sum of AMP. As ICP rises, the cumulative sum of AMP resembles exactly the Starling pressure-volume curve. The steepest slope point on the curve is the critical ICP (blue arrow). 


\section{The RAP Index}

The RAP index is the correlation coefficient between mean ICP and AMP (i.e., the correlation coefficient $[R]$ between AMP amplitude $[\mathrm{A}]$ and mean pressure $[\mathrm{P}])$. The RAP index has been shown to be a reliable measure of compensatory reserve [4] and is an accurate measure of intracranial compliance [5].

In the literature describing AMP and ICP and the cumulative sum of AMP and ICP (pressure-volume curve), the RAP index starts at 0 due to the lack of synchronous changes between the AMP and the mean ICP. This means that an increase in intracranial volume does not always result in an ICP rise. When ICP is contributing to increased AMP, the RAP index rises to +1 , and there is a synchronization between the rise in ICP and the rise in AMP. In terms of the cumulative sum of AMP and ICP (pressure-volume curve), a small rise in intracranial volume results in a larger rise in ICP. When the ICP continues to rise and breaches the critical ICP level, the cerebral compensatory reserve becomes exhausted. This means cerebral autoregulation has collapsed. At this point the AMP shows an inverse relationship to ICP, i.e., the rising ICP lead is associated with a decreasing AMP. So, the RAP index value becomes -1. It is at this point that cerebral ischemia-induced irreversible brain damage will likely occur and, ultimately, brain herniation. Finally, the ICP rises enough to not show brain pulsation and the heart beat no longer affects the ICP. The RAP index value becomes 0 again. Thus, we expect the critical ICP is the moment when the RAP index moves from positive to negative (Figure 1).

\section{MATERIALS AND METHODS}

\section{Patients and Data Collection}

We retrospectively reviewed the medical records of all the patients who underwent emergency surgery by the author (JSK) due to poor WFNS graded aneurysmal SAH at admission between January 2013 and December 2018 in Inje University Haeundae Paik Hospital. The institutional review board approval has been waived due to a retrospective nature of our study. Poor grade defined as WFNS grade V. The following data were collected and analyzed with regard to the age, sex, Glasgow coma scale (GCS), WFNS grade, postoperative mental status, and outcomes at 14-day follow-up. The 14-day outcome was adopted to limit direct deaths from cerebral hemorrhage. We analyzed 35 patients who were admitted due to a ruptured aneurysm and WFNS grade V. We placed an EVD or hematoma catheter after clipping for the purposes of draining CSF or the hematoma, thus controlling ICP. A total of seven patient ICPs were monitored. We placed an EVD catheter in three patients at Paine's point before aneurysmal clipping. We removed the sylvian hematoma first in two patients and then placed an indwelling drainage catheter in the sylvian fissure after aneurysmal clipping. One patient presented with a temporal base intracerebral hematoma (ICH). We put an indwelling drainage catheter at the hematoma, then removed the bed after ICH removal and aneurysmal clipping. For the other patient with subdural hematoma, we inserted a subdural type Integra Camino ICP probe (Integra LifeSciences Corporation, Plainsboro, NJ, USA) in the temporal cortex at the end of the operation. We also compared the mortality rate between patients who had ICP monitoring only and those who had both ICP monitoring and the RAP index.

\section{RESULTS}

We retrospectively studied 136 patients with ruptured cerebral aneurysm clipping from January 2013 to December 2018. Among 136 patients, 35 patients (25.9\%) were WFNS grade V on emergency room presentation with aneurysmal SAH and received emergent surgical treatment (Table 1). There were 19 females and 16 males, with a mean age of 59.2 years (range, 37 to 88 years). Thirteen patients were fully comatose (GCS, 3 ). The GCS score on admission ranged from 3 to 6 (mean, 4.3). We included patients with ruptured aneurysms who also had unruptured incidental aneurysms at the same time.

The in-hospital 14-day mortality (no self-respiration or brain stem signs, i.e., clinical brain death) was $48.5 \%(n=17)$. Five patients expired due to general medical complications including pneumonia and acute renal failure. Until 2017, eight of 22 poor WFNS graded SAH patients expired (36\%). In 2018, four of eight poor WFNS graded SAH patients expired (50\%). Seven patients had real-time ICP monitoring (Table 2). Before 2018, three of five poor WFNS graded SAH patients who had realtime ICP monitoring died (60\%). When the ICP monitor sent an alarm, immediate cerebral CT scans were obtained and revealed severe brain injury including ventricular collapse, severe swelling, and infarctions.

Fortunately, no death was reported for the two patients monitored with the RAP index in 2018. We did detect the RAP index and AMP changes from negative to positive over the period of ICP increase. This inflexion point can indicate critical ICP breaching and prompts immediate brain CT scan follow up and relevant additional management to decrease the ICP before the clinical worsening. As a result, we reduced irreversible damage to brain tissue and obtained better subsequent 
Table 1. Overall patient characteristics and results

\begin{tabular}{|c|c|c|c|c|c|c|c|c|}
\hline No. & Age (yr) & Sex & Aneurysm location & Hypertension & Smoking & GCS & 14-Day outcome & ICP monitor \\
\hline 1 & 44 & Male & A-com & Yes & Yes & 3 & $\mathrm{E}$ & \\
\hline 2 & 43 & Male & MCA & & & 4 & & \\
\hline 3 & 40 & Female & MCA & Yes & & 6 & & \\
\hline 4 & 85 & Female & P-com & Yes & & 5 & & \\
\hline 5 & 58 & Male & MCA & Yes & & 6 & M & \\
\hline 6 & 37 & Male & A-com & & Yes & 3 & $\mathrm{E}$ & Yes \\
\hline 7 & 65 & Female & MCA & Yes & & 5 & & \\
\hline 8 & 88 & Female & $\mathrm{MCA}, \mathrm{M} 1^{\mathrm{a}}$ & Yes & & 5 & & Yes \\
\hline 9 & 66 & Female & $\mathrm{M} 2, \mathrm{M}^{\mathrm{a}}$ & Yes & & 5 & & \\
\hline 10 & 44 & Female & $\mathrm{A} 2$ & & & 5 & & \\
\hline 11 & 63 & Male & A-com & & & 3 & $E$ & \\
\hline 12 & 87 & Male & P-com & Yes & Yes & 4 & $M$ & \\
\hline 13 & 41 & Male & A-com & Yes & Yes & 3 & $\mathrm{E}$ & Yes \\
\hline 14 & 52 & Male & A-com & & Yes & 3 & $\mathrm{E}$ & \\
\hline 15 & 49 & Female & A-com & Yes & & 3 & $\mathrm{E}$ & \\
\hline 16 & 66 & Female & A-com & Yes & & 5 & M & \\
\hline 17 & 76 & Female & ICA & Yes & Yes & 6 & & Yes \\
\hline 18 & 50 & Male & MCA & Yes & Yes & 3 & $\mathrm{E}$ & Yes \\
\hline 19 & 62 & Male & MCA & Yes & & 6 & & \\
\hline 20 & 64 & Male & MCA & Yes & Yes & 3 & $E$ & \\
\hline 21 & 59 & Female & VA dissecting & Yes & & 3 & $\mathrm{E}$ & \\
\hline 22 & 69 & Female & MCA & Yes & & 5 & $\mathrm{E}$ & \\
\hline 23 & 55 & Male & MCA & & & 6 & $\mathrm{E}$ & \\
\hline 24 & 48 & Male & ICA & & Yes & 3 & $E$ & \\
\hline 25 & 77 & Male & MCA & Yes & Yes & 3 & M & \\
\hline 26 & 51 & Female & MCA & Yes & & 3 & & Yes \\
\hline 27 & 66 & Female & A-com & Yes & & 6 & & \\
\hline 28 & 48 & Male & MCA & Yes & Yes & 4 & & Yes \\
\hline 29 & 62 & Female & P-com & Yes & & 4 & & \\
\hline 30 & 40 & Female & P-com & & & 5 & & \\
\hline 31 & 63 & Female & P-com & Yes & Yes & 5 & & \\
\hline 32 & 65 & Female & P-com & Yes & & 3 & & \\
\hline 33 & 50 & Female & A-com & Yes & & 3 & $M$ & \\
\hline 34 & 80 & Male & A-com & Yes & Yes & 6 & & \\
\hline 35 & 62 & Female & P-com & Yes & & 6 & & \\
\hline
\end{tabular}

GCS: Glasgow coma scale; ICP: intracranial pressure; A-com: anterior communicating artery aneurysm; MCA: middle cerebral artery aneurysm; P-com: posterior communicating artery aneurysm; ICA: internal carotid artery; VA: vertebral artery; E: expired due to brain lesion; M: expired due to general medical condition.

${ }^{2}$ The patients had both a ruptured aneurysm and unruptured incidental aneurysms at the same time.

improvement in patient outcomes.

Illustrated Case: Patient No. 26

A 51-year-old woman presented as comatose due to rupture of a right MCA bifurcation aneurysm. A preoperative computed tomography (CT) scan demonstrated SAH and a small amount of subdural hematoma (Figure 2A). She had undergone craniotomy and clipping of the MCA aneurysm (Figure 2B). During 
Table 2. Summary of data in seven patients with ICP monitor

\begin{tabular}{|c|c|c|c|c|c|}
\hline Patient No. & Type of ICP monitor & Preoperative CT & Duration of ICP monitoring & Follow-up CT decision ${ }^{\mathrm{a}}$ & 14-Day GOS score \\
\hline 6 & EVD catheter & IVH & 5 & ICP & Expire \\
\hline 8 & Hematoma catheter & Sylvian hematoma & 10 & ICP & 3 \\
\hline 13 & EVD catheter & IVH & 8 & ICP & Expire \\
\hline 17 & EVD catheter & IVH & 13 & ICP & 2 \\
\hline 18 & Hematoma catheter & Temporal hematoma & 4 & ICP & Expire \\
\hline 26 & Camino & Subdural hematoma & 8 & ICP, RAP & 2 \\
\hline 28 & Hematoma catheter & Sylvian hematoma & 2 & ICP, RAP & 4 \\
\hline
\end{tabular}

ICP: intracranial pressure; CT: computed tomography; GOS: Glasgow outcome scale; EVD: extraventricular drainage; IVH: intraventricular hemorrhage; RAP: RAP index.

aMain reason for predicting changes in patient status to perform a follow-up CT scan.
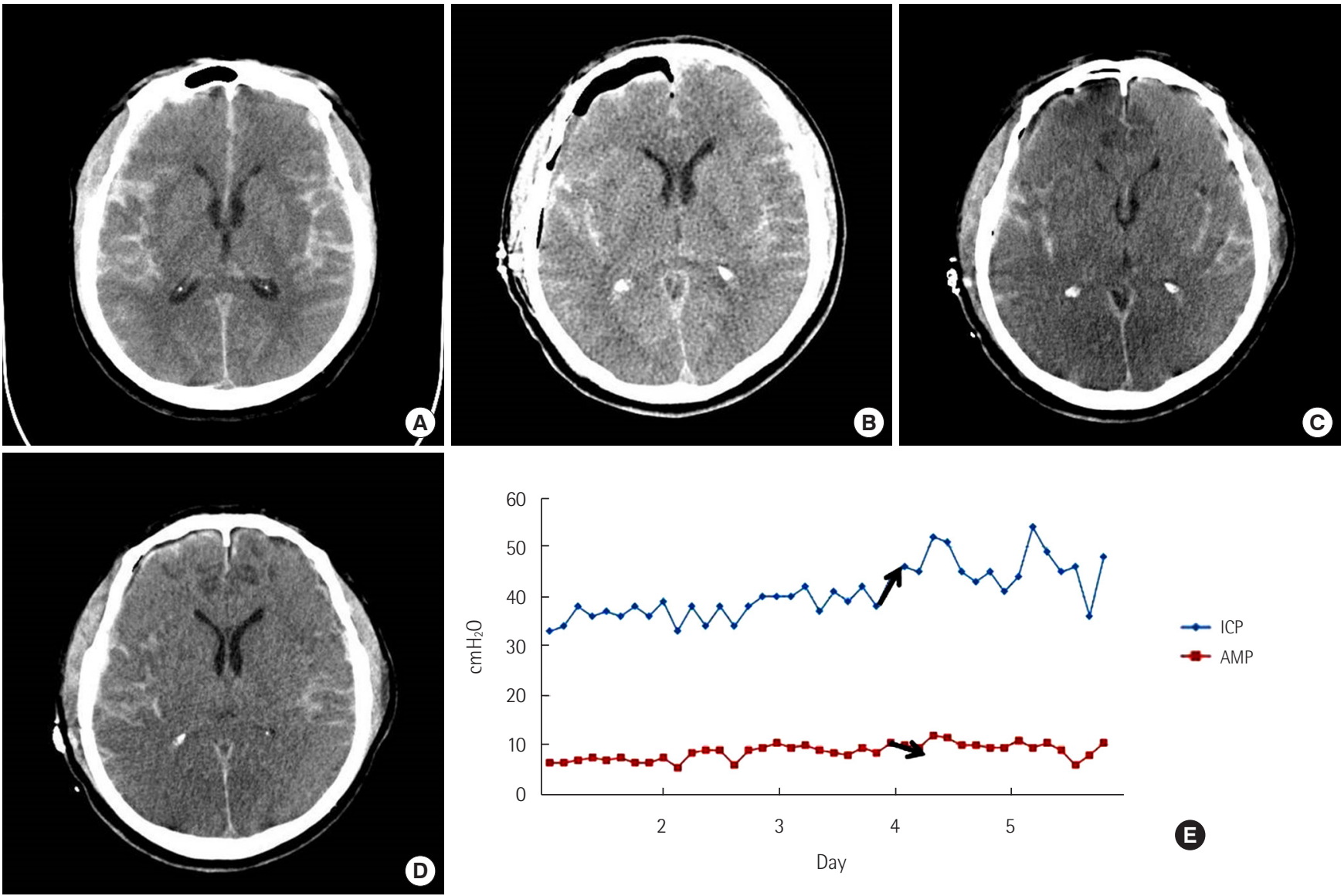

Figure 2. A 51-year-old woman presented as comatose due to rupture of a right middle cerebral artery aneurysm bifurcation aneurysm. (A) A preoperative computed tomography (CT) scan shows subarachnoid hemorrhage and a small amount of subdural hematoma. (B) Immediate postoperative CT scan shows craniotomy state and pneumocephalus. (C) The 4th postoperative day's CT scan shows severe brain swelling and diffuse low-density lesion. (D) The 7th postoperative day's CT scan shows slightly improving brain swelling and a nonprogressing low-density lesion. (E) Illustration graph of the intracranial pressure (ICP) and amplitude (AMP) of the patient. On the 2nd postoperative day, the ICP was still over $30 \mathrm{cmH}_{2} \mathrm{O}$ but the AMP was not significantly changed. However, on the 4th postoperative day, the ICP rose over $40 \mathrm{cmH}_{2} \mathrm{O}$ and AMP decreased (black arrows). We thought the RAP index was changing toward a negative aspect and the patient had reached critical ICP.

the operation we inserted a subdural type Integra Camino ICP probe on the temporal cortex. Immediate postoperative ICP was $30 \mathrm{cmH}_{2} \mathrm{O}$ (the initial ICP). Her mental status did not improve except for some self-respiration. On the second postop- 
erative day, the ICP was still over $30 \mathrm{cmH}_{2} \mathrm{O}$ but the AMP was not significantly changed. However, on the 4 th postoperative day, the ICP rose over $40 \mathrm{cmH}_{2} \mathrm{O}$ and the AMP decreased (Figure $2 \mathrm{E}$ ). We thought the RAP index was moving toward negative and the patient had reached critical ICP. Immediate CT scan revealed brain swelling and scattered low density lesions but, this time, without a specific mass effect lesion that needed to be decompressed (Figure 2C). We decided to apply multiple modalities to decrease the ICP by increasing mannitol dosing, sedation, and initiating a hypothermia procedure. After a while, the patient's ICP began to decrease but her AMP was not changed. On the 7th postoperative day, we did another CT scan and decided to remove the subdural ICP probe (Figure 2D).

\section{Illustrated Case: Patient No. 28}

A 48-year-old man presented in a semicomatose state due to left MCA bifurcation aneurysm rupture. A preoperative CT scan demonstrated SAH and a thick sylvian hematoma (Figure 3A). He had undergone craniotomy and clipping for the MCA aneurysm (Figure 3B). We inserted a drainage catheter into the sylvian hematoma site to monitor ICP. Immediate postoperative ICP was checked and was still over $30 \mathrm{cmH}_{2} \mathrm{O}$, which was recorded as the initial ICP. Luckily, his mental status recovered to deep drowsiness and his right-side motor power grade was III. On the second postoperative day, although his neurologic status was not aggravated, the ICP rose from 30 $\mathrm{cmH}_{2} \mathrm{O}$ to $40 \mathrm{cmH}_{2} \mathrm{O}$ and the AMP decreased remarkably (Figures $3 \mathrm{E}, \mathrm{F}$, and 4). We thought the RAP index was beginning to change into a negative status. Immediate CT scan showed an
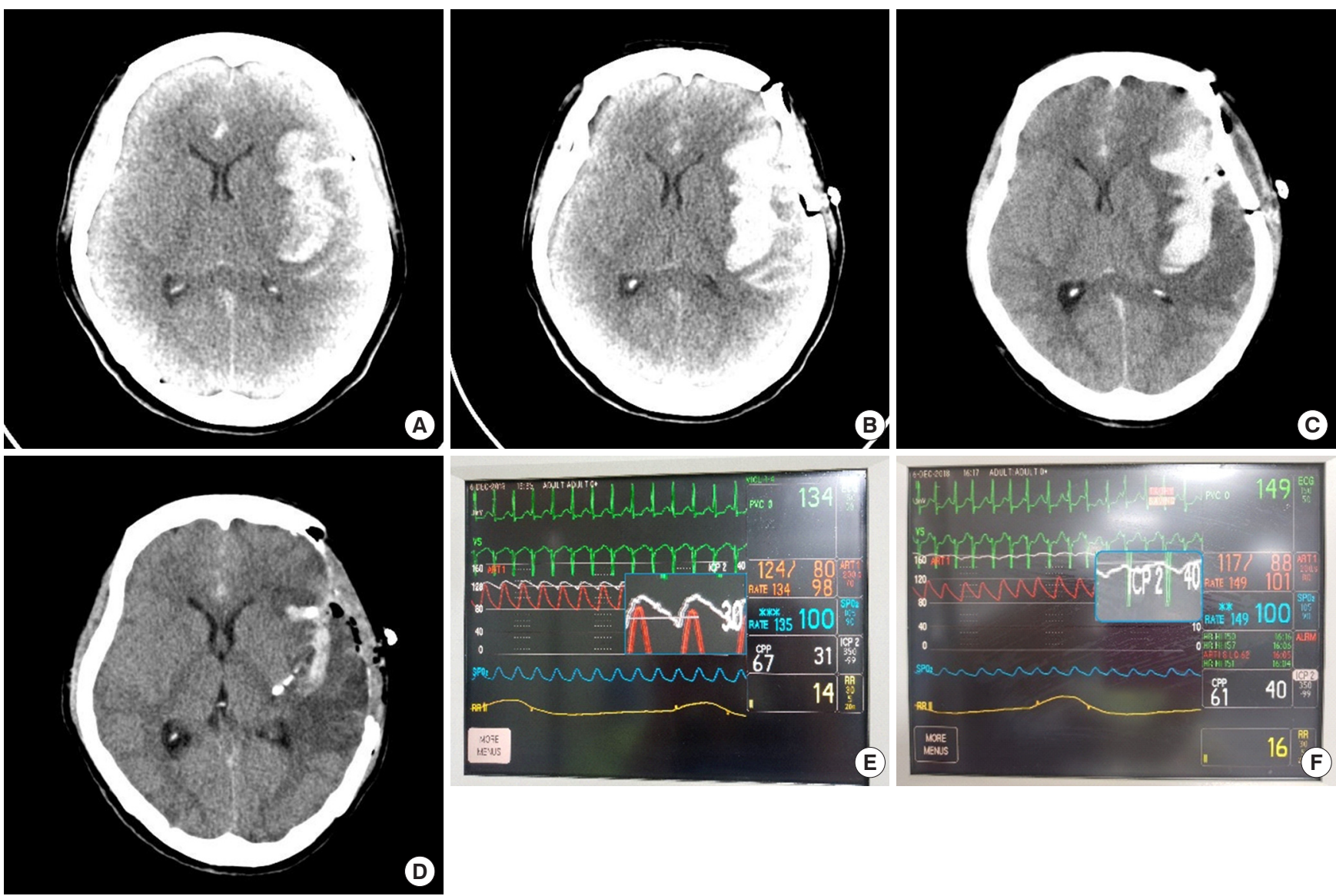

Figure 3. A 48-year-old man presented in a semicomatose state due to the rupture of a left middle cerebral artery aneurysm bifurcation aneurysm. (A) A preoperative computed tomography (CT) scan shows subarachnoid hemorrhage (SAH) and a thick sylvian hematoma. (B) Immediate postoperative CT scan shows craniotomy and remnant SAHs. (C) The 2nd postoperative day's CT scan shows an increased lowdensity lesion around the sylvian hematoma. (D) After the second operation, the CT scan shows craniectomy state and decreased brain swelling and sylvian hematoma. (E) Intracranial pressure (ICP) monitoring using a sylvian drainage catheter connected to an arterial pressure monitor kit. (F) On the 2nd postoperative day, ICP rose to $40 \mathrm{~cm} \mathrm{H}_{2} \mathrm{O}$ from $30 \mathrm{cmH}_{2} \mathrm{O}$ and amplitude decreased remarkably. The RAP index just changed negatively implying the patient has reached a critical ICP (blue square, white line). 


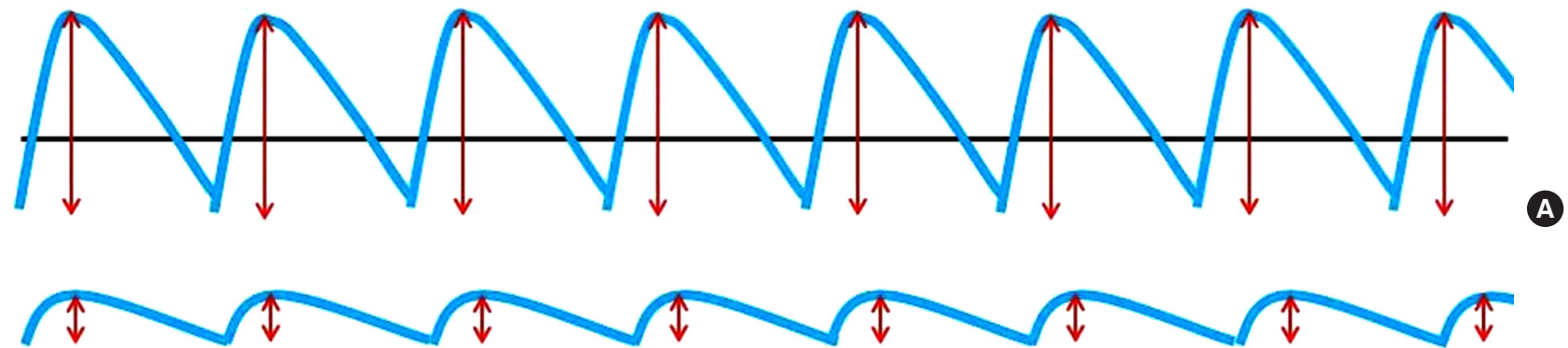

Figure 4. Intracranial pressure (ICP) monitoring waves depicting amplitude (AMP) changes from a positive RAP index to a negative one as the patient ICP fluctuates. (A) Red lines illustrate the AMP of the ICP wave (blue line) and the average of the AMPs gives the ICP mean pulse AMP. (B) Decreased AMP implies the RAP index inversion from the positive to the negative which prompted additional management for the patient just reaching critical ICP.

increased low-density lesion around the sylvian hematoma which was exerting a radial mass effect (Figure 3C). We decided on a second operation. This time, for craniectomy and removal of the sylvian hematoma (Figure 3D). After the operation, his ICP dropped under $20 \mathrm{cmH}_{2} \mathrm{O}$ and the AMP was restored.

\section{DISCUSSION}

The reason for monitoring the ICP is to track cerebral blood flow (CBF). Cerebrovascular reserve refers to the capacity of the brain to increase cerebral blood volume to maintain a constant regional $\mathrm{CBF}(\mathrm{rCBF})$ in the face of low cerebral perfusion pressure (CPP). $\mathrm{rCBF}$ is determined by the ratio of $\mathrm{CPP}$ to cerebrovascular resistance using the following formula $[6,7]$.

$\mathrm{rCBF}=\mathrm{CPP} /$ vascular resistance

$\mathrm{CPP}$ is related to systemic mean arterial pressure (MAP) and ICP by the equation: $\mathrm{CPP}=\mathrm{MAP}-\mathrm{ICP}$

Under normal circumstances, a MAP between 60 to 160 $\mathrm{mmHg}$ and an ICP of about $10 \mathrm{mmHg}$ (CPP of 50-150 mmHg) can be maintained with autoregulation $[8,9]$. The monitoring of ICP is of great clinical importance in order to surveil any changes in patient increased intracranial pressure (IICP) to predict onset of critical ICP that might call for an additional surgical procedure. At an ICP of $25 \mathrm{mmHg}$, aggressive treatment is started in most intensive care units. The ICP is dynamic because it can be changed by the patient's position and blood pressure, and the ventilator mode, as well as the calibration of the measuring device. The unique capacity of each patient's brain autoregulation may also vary. Thus, the monitored numeric ICP pressure alone is not sufficient to predict each patient's critical ICP. Especially, in patients with poor graded $\mathrm{SAH}$, we may miss precisely timing postoperative surgical treatment if we only rely on ICP monitoring. In addition, with patients who initially present as comatose or semicomatose, it is not always possible to catch neurologic deterioration. After we lost patients whose postoperative neurologic monitoring was checked only via their ICP, we came to realize that their critical ICPs had been missed as well as the right time to launch any relevant treatment plan. We needed an additional way to more precisely monitor patients. The RAP index was designed for the purpose of predicting critical ICP. It was designed as the coefficient between the mean ICP and the mean ICP pulse AMP and can function as a potential marker of neurological deterioration in traumatic brain injury and hydrocephalus patients $[4,10]$. We can detect changes in the AMP and RAP index through ICP monitoring before the patient gets clinically worse. This allowed us to devise and launch an impromptu treatment plan for each patient. As a result, we could reduce irreversible damage to brain tissue and optimize subsequent improvement in patient outcomes [11].

The role of ICP monitoring for IICP patients is very important for improving patient outcomes. However, it is has been shown that ICP monitoring alone may not be the perfect option for early detection of neurologic deterioration, and thus providing better clinical outcomes. That being said, ICP monitoring reinforced with both the RAP index and AMP can provide important intracranial physiological data that indicate when a patient requires immediate medical attention. However, due to our small number of patients, this study is underpowered. Further studies on the role of the RAP index and AMP including ICP monitoring with a larger sample size of patients are warranted. 


\section{CONFLICT OF INTEREST}

No potential conflict of interest relevant to this article was reported.

\section{ORCID}

Sung-Chul Jin https://orcid.org/0000-0001-5282-9329

Byung Sam Choi https://orcid.org/0000-0002-8760-9593

Jung-Soo Kim

\section{AUTHOR CONTRIBUTIONS}

Conceptualization: JSK. Data curation: JSK. Formal analysis: BSC. Methodology: JSK. Project administration: JSK. Visualization: BSC. Writing - original draft: SCJ. Writing - review \& editing: SCJ.

\section{REFERENCES}

1. Hoogmoed J, Coert BA, van den Berg R, Roos YB, Horn J, Vandertop WP, et al. Early treatment decisions in poor-grade patients with subarachnoid hemorrhage. World Neurosurg 2018; 119:e568-73.

2. Zheng K, Zhao B, Tan XX, Li ZQ, Xiong Y, Zhong M, et al. Comparison of aggressive surgical treatment and palliative treatment in elderly patients with poor-grade intracranial aneurysmal subarachnoid hemorrhage. Biomed Res Int 2018;2018: 5818937.
3. Aguilar-Aguilar G, García-Raimundo M, Talamantes-Escribá F, Maruenda-Paulino A, Badenes-Catalá R, Belda-Nacher FJ. Intraparenchymal cerebral hematoma following the insertion of a catheter for monitoring intracranial pressure. Rev Esp Anestesiol Reanim 1999;46:45-6.

4. Czosnyka M, Steiner L, Balestreri M, Schmidt E, Smielewski P, Hutchinson PJ, et al. Concept of "true ICP" in monitoring and prognostication in head trauma. Acta Neurochir Suppl 2005; 95:341-4.

5. Varsos GV, Kasprowicz M, Smielewski P, Czosnyka M. Modelbased indices describing cerebrovascular dynamics. Neurocrit Care 2014;20:142-57.

6. Powers WJ. Cerebral hemodynamics in ischemic cerebrovascular disease. Ann Neurol 1991;29:231-40.

7. Momjian-Mayor I, Baron JC. The pathophysiology of watershed infarction in internal carotid artery disease: review of cerebral perfusion studies. Stroke 2005;36:567-77.

8. Steiner LA, Andrews PJ. Monitoring the injured brain: ICP and CBF. Br J Anaesth 2006;97:26-38.

9. Duschek S, Schandry R. Reduced brain perfusion and cognitive performance due to constitutional hypotension. Clin $\mathrm{Au}$ ton Res 2007;17:69-76.

10. Kim DJ, Czosnyka Z, Keong N, Radolovich DK, Smielewski P, Sutcliffe MP, et al. Index of cerebrospinal compensatory reserve in hydrocephalus. Neurosurgery 2009;64:494-501.

11. Hall A, O'Kane R. The best marker for guiding the clinical management of patients with raised intracranial pressure-the RAP index or the mean pulse amplitude? Acta Neurochir (Wien) 2016;158:1997-2009. 